\title{
Neutrino oscillation and CPT violation due to quantum decoherence at DUNE
}

\author{
F. N. Díaz ${ }^{* \dagger}$ \\ Pontificia Universidad Católica del Perú \\ E-mail: felix.diaz@pucp.edu.pe \\ J. C. Carrasco-Martínez \\ Pontificia Universidad Católica del Perú \\ E-mail: juan.carrasco@pucp.edu.pe
}

A. M. Gago $§$

Pontificia Universidad Católica del Perú

E-mail: agago@pucp.edu.pe

\begin{abstract}
In this work we study the intrinsic CPT violation in the neutrino oscillations phenomena produced by quantum decoherence as sub-leading effect. In the usual density matrix representation, we find that only fifteen elements of the decoherence matrix violate the CPT symmetry intrinsically. We find exact solutions for the CPT asymmetry function in vacuum. We define an observable to make predictions of this model for the future Long-Baseline experiment, DUNE. We found values of the decoherence parameters with $5 \sigma$ of discrepancy to standard physics which are allowed by the current experimental limits, suggesting hints for new physics by this model in the context of future experiments.
\end{abstract}

European Physical Society Conference on High Energy Physics - EPS-HEP2019 -

10-17 July, 2019

Ghent, Belgium

\footnotetext{
* Speaker.

${ }^{\dagger}$ Grant 000236-2015-FONDECYT-DE.

$\ddagger$ Grant No. 233-2015-2-CONCYTEC-FONDECYT.

${ }^{\S}$ Dirección de Gestión de la Investigación at PUCP, grant DGI-2017-3-0019.
} 


\section{Introduction}

Although the phenomenon of neutrino oscillation is quite corroborated [1,2, 3], new physics can be manifested as sub-leading effect $[4,5,6,7,8]$. There are many candidates that can coexist with the standard oscillation, for instance, quantum decoherence in oscillation $[9,10]$. Moreover, on some occasions the new physics can produce a violation of the fundamental symmetries [11, 12].

On the other hand, the CPT theorem guarantes that the simultaneous transformations of $\mathrm{C}$ (charge conjugation), $\mathrm{P}$ (parity) and $\mathrm{T}$ (time reversal) is a fundamental symmetry of physical laws [13]. However, altough there is not experimental evidence for violation of this symmetry, theoretically there are ways to breakdown CPT in the context of physics Beyond the Standard Model. In this work, we study the theoretical framework of CPTV produced by quantum decoherence and propose a way to measure it in the context of the DUNE experiment.

\section{Quantum decoherence}

The time evolution of the neutrino that is considered as an open quantum system, i.e., a quantum system that interact with an unknown environment, is given by the Lindblad equation

$$
\frac{\partial \hat{\rho}(t)}{\partial t}=-i[\hat{H}, \hat{\rho}(t)]+\mathscr{D}[\hat{\rho}(t)]
$$

where $\hat{\rho}(t)$ is the neutrino density matrix, $\hat{H}$ is the hamiltonian of the system and $\mathscr{D}$ is the dissipative term that represents the decoherence phenomena.

Considering the 3 flavors, the general expression for probability transition $v_{\alpha} \rightarrow v_{\beta}$ is given by

$$
P_{v_{\alpha} \rightarrow v_{\beta}}=\frac{1}{N}+\frac{1}{2} \sum_{i, j} \rho_{i}^{\beta} \rho_{j}^{\alpha}\left[e^{M t}\right]_{i j},
$$

where $M=H+D$. In general, for the antineutrino case we have to make the replacement of coefficients $\rho \rightarrow \bar{\rho}$, hamiltonian matrices $H \rightarrow \bar{H}$ and decoherence matrices $D \rightarrow \bar{D}$. However, in this study we assume the same decoherence matrix for neutrino and antineutrino. From now, we will work in the vacuum mass eigenstates basis.

The coefficients of the flavor $\alpha$ neutrino matrix density are

$$
\begin{aligned}
& \rho_{0}^{\alpha}=\sqrt{2 / 3} \\
& \rho_{1}^{\alpha}=2 \operatorname{Re}\left(U_{\alpha 1}^{*} U_{\alpha 2}\right) \\
& \rho_{2}^{\alpha}=-2 \operatorname{Im}\left(U_{\alpha 1}^{*} U_{\alpha 2}\right) \\
& \rho_{3}^{\alpha}=\left|U_{\alpha 1}\right|^{2}-\left|U_{\alpha 2}\right|^{2} \\
& \rho_{4}^{\alpha}=2 \operatorname{Re}\left(U_{\alpha 1}^{*} U_{\alpha 3}\right) \\
& \rho_{5}^{\alpha}=-2 \operatorname{Im}\left(U_{\alpha 1}^{*} U_{\alpha 3}\right) \\
& \rho_{6}^{\alpha}=2 \operatorname{Re}\left(U_{\alpha 1}^{*} U_{\alpha 3}\right) \\
& \rho_{7}^{\alpha}=-2 \operatorname{Im}\left(U_{\alpha 2}^{*} U_{\alpha 3}\right) \\
& \rho_{8}^{\alpha}=\frac{1}{\sqrt{3}}\left(\left|U_{\alpha 1}\right|^{2}+\left|U_{\alpha 2}\right|^{2}-2\left|U_{\alpha 3}\right|^{2}\right)
\end{aligned}
$$

For antineutrino, we replace all the elements of PMNS matrix by their complex conjugates, i.e. $U_{i j} \rightarrow U_{i j}^{*}$. Then, we have the same coefficients when they are real $\left(\rho_{1}^{\alpha}, \rho_{3}^{\alpha}, \rho_{4}^{\alpha}, \rho_{6}^{\alpha}, \rho_{8}^{\alpha}\right)$ and negative when they are imaginary $\left(\rho_{2}^{\alpha}, \rho_{5}^{\alpha}, \rho_{7}^{\alpha}\right)$. 


\section{CPT Violation}

In order to examine the effects of a possible CPT violation originated by dissipation, we will use the decoherence oscillation probabilities formulas for vacuum, [10] . Even though we will work in the context of DUNE experiment, the use of vacuum formulas is well justified since this article is devoted to study these effects comparing the $v_{\mu}$ and $\bar{v}_{\mu}$ disappearance channels, where the matter effects are unimportant. Therefore, we have defined the following asymmetry, written for a generic $v_{\alpha}$ :

$$
\mathscr{A}_{\mathrm{CPT}}=P_{v_{\alpha} \rightarrow v_{\alpha}}-P_{\bar{v}_{\alpha} \rightarrow \bar{v}_{\alpha}},
$$

With the purpose of simplifying the analysis of the expression [3.1], we solve the oscillation probabilities considering the following hypotheses : the diagonal elements of the decoherence matrix $D$ are all equal to $\Gamma$, the dissipative matrix for neutrinos is equal to the corresponding for antineutrinos, $D=\bar{D}$, and the $D$ matrix is containing a single one non-diagonal elements at a time. We observe that, for having non null CPT violation, in general, we require, besides to have an specific non-zero $\beta_{i j}$, a non-zero value of $\delta_{C P}$, then, the asymmetry $\mathscr{A}_{\text {CPT }}$ depends on $\rho_{i} \rho_{j}$ terms. This dependency is explained by the change of sign of $\rho_{i} \rho_{j}$ when we switch to the antineutrino case $\left(\rho_{i} \rho_{\bar{j}}=-\rho_{i} \rho_{j}\right)$ a new term appears in the subtraction of probabilities. Therefore, we find that, in order to violate the CPT symmetry, one coefficient of the factor $\rho_{i} \rho_{j}$ must be $\rho_{2}$ or $\rho_{5}$ or $\rho_{7}$ and the other should be anyone of the others $\rho_{1}, \rho_{3}, \rho_{4}, \rho_{6}, \rho_{8}$ having in total fifteen cases.

Based on the similarities of the structure of the form for $\mathscr{A}_{\mathrm{CPT}}$ we can divide these fifteen cases into two groups, each group related to different set of $\beta_{i j}$, that we present at follows.

\subsection{Group 1}

The $\mathscr{A}_{\mathrm{CPT}}$ expression for the first group is given by:

$$
\mathscr{A}_{\mathrm{CPT}}=2 \beta_{i j} \frac{\sin \left[\Omega_{\beta_{i j}} t\right]}{\Omega_{\beta_{i j}}} \rho_{i}^{\alpha} \rho_{j}^{\alpha} e^{-\Gamma t},
$$

where $\Omega_{\beta_{i j}}=\sqrt{\Delta_{\beta_{i j}}{ }^{2}-\beta_{i j}^{2}}$, with $\Delta_{\beta_{i j}}=\Delta m_{\beta_{i j}}^{2} / 2 E$. This formula applies for nine $\beta_{i j}$, the details are given in Table 1 and Fig. 1.

$$
\begin{array}{c|c}
(i, j) & \Delta_{\beta_{i j}} \\
\hline(1,2),(2,3),(2,8) & \Delta_{12} \\
(4,5),(5,3),(5,8) & \Delta_{13} \\
(6,7),(7,3),(7,8) & \Delta_{23}
\end{array}
$$

Table 1: Here it is displayed each pair of indexes $(i, j)$, which corresponds to a one of the nine $\beta_{i j}$. The $(i, j)$ in the same row are associated to a unique $\Delta_{\beta_{i j}}$

\subsection{Group 2}

The asymmetry for the parameters $\beta_{i j}: \beta_{15}, \beta_{24}, \beta_{17}, \beta_{26}, \beta_{47}$ and $\beta_{56}$ is proportional to $\rho_{i}^{\alpha} \rho_{j}^{\alpha}$ and $\rho_{k}^{\alpha} \rho_{l}^{\alpha}$. For the indices $i j$, we have a specific $k l$, and these indices are associated with a determined mass squared diference value, for the complete details see Table 2 . The six expressions for 


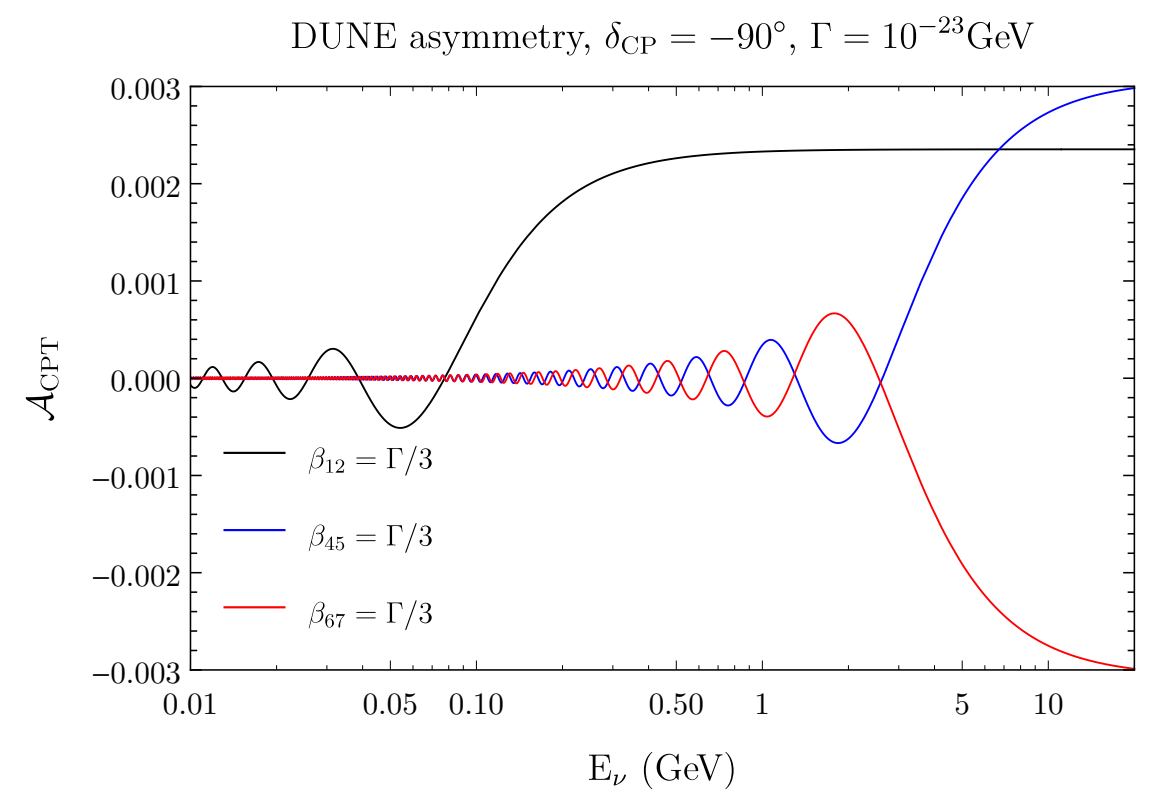

Figure 1: Group 1, asymmetry as a function on the energy. We consider all the diagonal elements equal to $\Gamma$ and one $\beta_{i j}$ at a time.

the CPTV formula are obtained per each pair $i j, k l$ plus exchanging $i j \leftrightarrow k l$, with all its correspondent terms associated with them. The explicit formula is given by:

$$
\begin{aligned}
& \mathscr{A}_{\mathrm{CPT}}=\beta_{i j} \frac{1}{\sqrt{\Omega^{4}-4 \Delta_{\beta_{i j}}^{2} \Delta_{\beta_{k l}}^{2}}} \\
& \times\left[\left(\Omega_{+} \sin \Omega_{+} t-\Omega_{-} \sin \Omega_{-} t\right) \rho_{i}^{\alpha} \rho_{j}^{\alpha}\right. \\
& \left.-\Delta_{\beta_{i j} \Delta_{\beta_{k l}}}\left(\frac{\sin \Omega_{+} t}{\Omega_{+}}-\frac{\sin \Omega_{-} t}{\Omega_{-}}\right) \rho_{k}^{\alpha} \rho_{l}^{\alpha}\right] e^{-\Gamma t} .
\end{aligned}
$$

where $\Omega^{2}=\Delta_{\beta_{i j}}^{2}+\Delta_{\beta_{k l}}^{2}-\beta_{i j}^{2}$ and $\Omega_{ \pm}=\frac{1}{\sqrt{2}} \sqrt{\Omega^{2} \pm \sqrt{\Omega^{4}-4 \Delta_{\beta_{i j}}^{2} \Delta_{\beta_{k l}}^{2}}}$.

$$
\begin{aligned}
\left\{(i, j), \Delta_{\beta_{i j}}\right\} \leftrightarrow\left\{(k, l), \Delta_{\beta_{k l}}\right\} \\
\hline\left\{(1,5), \Delta_{12}\right\} \leftrightarrow\left\{(2,4), \Delta_{13}\right\} \\
\left\{(1,7), \Delta_{12}\right\} \leftrightarrow\left\{(2,6), \Delta_{23}\right\} \\
\left\{(4,7), \Delta_{13}\right\} \leftrightarrow\left\{(5,6), \Delta_{23}\right\}
\end{aligned}
$$

Table 2: Here it is shown how is the relation between the six indexes $(i, j)$ and $(k, l)$, each of them associated to its corresponding $\beta$ and its neutrino mass square differences.

\section{DUNE Experiment}

The DUNE experiment is one of the most powerful tool designed for studying fundamental (and unknown) aspects of neutrino physics, among others, the measurement of the leptonic CP violation phase $\delta_{C P}$, the mass hierarchy, solving the $\theta_{23}$ octant degeneracy, etc. It also has very good 


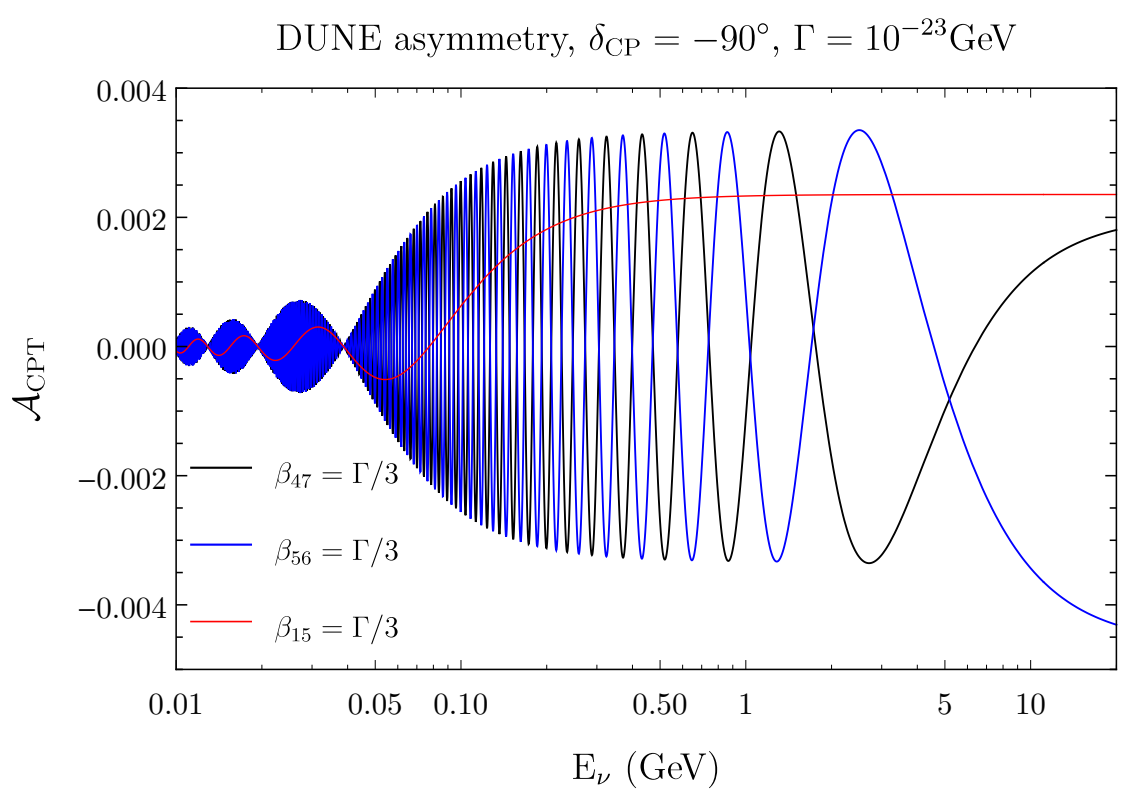

Figure 2: Group 2, asymmetry as a function on the energy. We consider all the diagonal elements equal to $\Gamma$ and one $\beta_{i j}$ at a time.

\begin{tabular}{c|c} 
Parameter & Value \\
\hline$\theta_{12}$ & $33.63^{\circ}$ \\
$\theta_{13}(\mathrm{NH})$ & $8.52^{\circ}$ \\
$\theta_{23}(\mathrm{NH})$ & $48.7^{\circ}$ \\
$\Delta m_{21}^{2}$ & $7.4 \times 10^{-5} \mathrm{eV}^{2}$ \\
$\Delta m_{31}^{2}(\mathrm{NH})$ & $2.515 \times 10^{-3} \mathrm{eV}^{2}$ \\
Baseline & $1300 \mathrm{Km}$
\end{tabular}

Table 3: Values for standard oscillation parameters.

capabilities for catching a relevant number of supernova neutrinos and for doing proton decay science. Moreover, DUNE would be able to test many possibilities of beyond standard model physics that can be manifested through neutrino oscillation. The experiment will consist in a muon neutrino (antineutrino) beam traveling through the Earth $1300 \mathrm{~km}$, from Fermilab to Sanford Underground Research Facility (SURF) with the average matter density of $\rho_{\text {DUNE }}=2.96 \mathrm{~g} / \mathrm{cm}^{3}$.

We assume the configuration of $80 \mathrm{GeV}$ energy with $1.07 \mathrm{MW}$ power in the primary proton beam from the Main Injector runing over 5 years for exposure for each mode (FHC and RHC). The neutrino beam, resulting from the proton beam, are expected to be detected in a massive liquid argon time-projection chamber (LArTPC) that consists of 40 Kton of liquid argon. For simulating DUNE, we use GLoBES $[14,15]$ taking the information for the cross section, neutrino fluxes, resolution function and efficiency given by the collaboration [16]. While, the matter neutrino oscillation probabilities plus decoherence was calculated with nuSQuIDS [17]. 


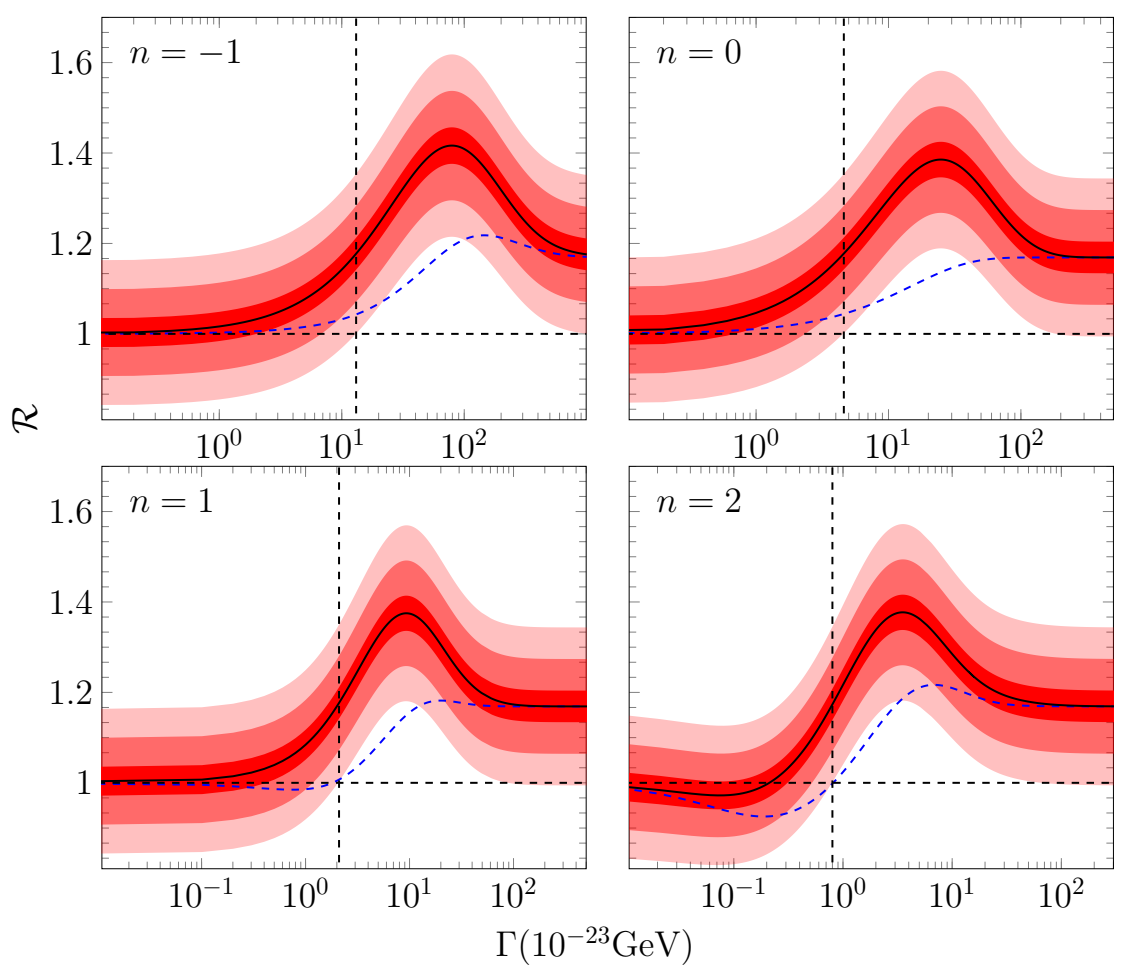

Figure 3: The black horizontal dashed line is the expected in the standard oscillation. The blue dashed line corresponds only to the diagonal decoherence matrix. Meanwhile, the solid black line corresponds to the case of a non diagonal decoherence matrix, both cases evaluated at $\delta_{C P}=3 \pi / 2$. The red fringes (small, medium and large) represent the statistical error $1 \sigma, 3 \sigma$ and $5 \sigma$ (respectively). The $\beta$ 's used corresponds to $\beta_{28}=\Gamma / \sqrt{3}, \beta_{12}=(\sqrt{2 / 3}) \Gamma / 3$ and $\beta_{56}=-\beta_{47}=\Gamma / 3$. The intersection between the black horizontal dashed line with the vertical one marks the $5 \sigma$ significance of the non diagonal decoherence matrix case relative to the standard oscillation case.

In order to test the CPTV effects we define the following experimental observable:

$$
\mathscr{R}=\frac{\Delta N^{\mathrm{std} \oplus \text { deco }}}{\Delta N^{\mathrm{std}}},
$$

where $\Delta N^{\operatorname{std}(\operatorname{std} \oplus \text { deco })}=N_{v_{\mu}}-N_{\bar{v}_{\mu}}$ is the difference between the total events rates for neutrino and antineutrino, respectively, where $\operatorname{std}(\operatorname{std} \oplus$ deco) stands for standard oscillation (standard oscillation plus decoherence). The total event rates has been calculated in accordance with the CDR configuration [18], integrated from 0.5 and $20 \mathrm{GeV}$, taking into account signal and background. Thus, when decoherence is absent we have $\mathscr{R}=1$. From now on, we focus on a special decoherence matrix that maximizes CPTV, we simultaneously turn on $\beta_{28}, \beta_{12}, \beta_{56}$ and $\beta_{47}$ in the following values: $\beta_{28}=\Gamma / \sqrt{3}, \beta_{12}=(\sqrt{2 / 3}) \Gamma / 3$ and $\beta_{56}=-\beta_{47}=\Gamma / 3$. On the other hand, the decoherence parameters could be energy dependent, therefore, we will consider this dependence as follows: $\Gamma(E)=\Gamma(E / \mathrm{GeV})^{n}$ for $n=-1,0,1,2$.

In Fig. 3, we present the observable $\mathscr{R}$, with its corresponding error bands for $1 \sigma, 3 \sigma$ and $5 \sigma$, versus $\Gamma$, for $n=-1,0,1$, and 2 . We have that for the non diagonal decoherence case a $5 \sigma$ discrepancy, respect to the expectation value for standard oscillation $(\mathscr{R}=1)$, is reached at the following $\Gamma=\{13.1,4.6,2.1,0.8\} \times 10^{-23} \mathrm{GeV}$ for $n=-1,0,1$ and 2 , respectively. 


\section{Conclusions}

We have obtained a breakdown of the CPT symmetry as a result of considering that the neutrino system is affected by the environment. Moreover, we have studied and proposed the possibility of measuring CPTV through the $v_{\mu} \rightarrow v_{\mu}$ disappearance channel and our observable $\mathscr{R}$, in the context of DUNE experiment.

Considering different energy dependencies of the decoherence parameter, $n=-1,0,1$, and $2, \delta_{C P}=3 \pi / 2$, and a non diagonal decoherence matrix, we achieve $5 \sigma$ of discrepancy for $\mathscr{R}$ with respect to the expectation value at the standard oscillation case, $\mathscr{R}=1$, for the following $\Gamma$ : $\{13.1,4.6,2.1,0.8\} \times 10^{-23} \mathrm{GeV}$, for $n=-1,0,1$ and 2 , respectively.

\section{References}

[1] S. Fukuda et al. [Super-Kamiokande Collaboration], Phys. Rev. Lett. 86, 5651 (2001)

[2] Q. R. Ahmad et al. [SNO Collaboration], Phys. Rev. Lett. 89, 011302 (2002)

[3] T. Kajita et al. [Super-Kamiokande Collaboration], Nucl. Phys. B 908, 14 (2016).

[4] J. M. Berryman, A. de Gouvêa, D. Hernández and R. L. N. Oliveira, Phys. Lett. B 742, 74 (2015)

[5] A. M. Gago, R. A. Gomes, A. L. G. Gomes, J. Jones-Perez and O. L. G. Peres, JHEP 1711, 022 (2017)

[6] R. Picoreti, M. M. Guzzo, P. C. de Holanda and O. L. G. Peres, Phys. Lett. B 761, 70 (2016)

[7] A. M. Gago, M. M. Guzzo, H. Nunokawa, W. J. C. Teves and R. Zukanovich Funchal, Phys. Rev. D 64, 073003 (2001)

[8] A. Esmaili and A. Y. Smirnov, JHEP 1306, 026 (2013)

[9] F. Benatti and R. Floreanini, Phys. Rev. D 64, 085015 (2001)

[10] A. M. Gago, E. M. Santos, W. J. C. Teves and R. Zukanovich Funchal, hep-ph/0208166.

[11] J. T. Pantaleone, A. Halprin and C. N. Leung, Phys. Rev. D 47, R4199 (1993)

[12] M. Bustamante, A. M. Gago and C. Pena-Garay, JHEP 1004, 066 (2010)

[13] N. E. Mavromatos, hep-ph/0504143.

[14] P. Huber, M. Lindner and W. Winter, Comput. Phys. Commun. 167, 195 (2005)

[15] P. Huber, J. Kopp, M. Lindner, M. Rolinec and W. Winter, Comput. Phys. Commun. 177, 432 (2007)

[16] T. Alion et al. [DUNE Collaboration], arXiv:1606.09550 [physics.ins-det].

[17] C. A. Argüelles Delgado, J. Salvado and C. N. Weaver, Comput. Phys. Commun. 196, 569 (2015)

[18] R. Acciarri et al. [DUNE Collaboration], arXiv:1512.06148 [physics.ins-det]. 